\title{
Formation Temperature Distribution of the Turonian- Maastrichtian Fika Shale Formation from Wireline Logs, in Part of Borno Basin, Northeastern Nigeria
}

\author{
Anthony John.Ilozobhie ${ }^{1, *}$ and Dominic Akam $\mathrm{Obi}^{2}$ \\ ${ }^{1}$ Physics Department, University of Calabar \\ Nigeria \\ ${ }^{2}$ Geology Department, University of Calabar \\ Nigeria \\ *Corresponding author's email: anthonyilozobhie [AT] gmail.com
}

\begin{abstract}
The distribution of Formation Temperature of the Fika Shale Formation within part of Borno basin from five wireline logs was investigated. The area under studied covered about 20 by 40 kilometre square of the total land mass of the basin. The Fika shale sequence were identified at $865 \mathrm{~m}$ to $1795 \mathrm{~m}$ in Kinasar well, $640 \mathrm{~m}$ to $1990 \mathrm{~m}$ in Krumta, 980m to $1620 \mathrm{~m}$ in Masu, $700 \mathrm{~m}$ to $2710 \mathrm{~m}$ in Tuma, $710 \mathrm{~m}$ to $2220 \mathrm{~m}$ in Wadi. The plot of the entire Formation Temperature of the study area reveals remarkable steep variations in temperature with increased range from $68^{\circ} \mathrm{C}$ to $128^{\circ} \mathrm{C}$ starting from the southern eastern region to the north western region. This is possibly due to substantial temperature enhancing effects of the underlying basement complex. Interestingly, it was also observed that the minimal temperature variation occurred approximately $2^{\circ} \mathrm{C} /$ meter across the field and this also lays credence to the fact that the notable subsurface geothermal variation may be recent events initiated by the near-surface magmatic intrusive events which may have had adverse effects on the overlying sedimentary cover. Furthermore, it was deduced that the probability of hydrocarbon accumulation is better in the south eastern region than the north western region although the steep temperature variation of $2^{\circ} \mathrm{C} /$ meter within some part of the study area may perhaps reduce this possibility.
\end{abstract}

Keywords---- wireline, sequence, basement, intrusive, geothermal and magmatic

\section{INTRODUCTION}

The Nigerian sector (Borno Basin) of the Chad Basin is known to provide possible source rocks, reservoir beds and structural and stratigraphic environment required for the formation, exploration and exploitation of hydrocarbon. However, there has been no successful record of commercial discovery of Hydrocarbon within the Borno Basin contrary to the success recorded in the Chad Basin by other neighboring countries that are part of this Chad Basin system despite the huge Capital that has been invested by Federal government of Nigeria. Currently, over 730 square kilometers of data been acquired and interpreted by IDSL, a subsidiary of NNPC, in partnership with BGP of China with little to show for it despite the huge resources deplored by the federal government of Nigeria thus there is a need to critically re assess the potential of the basin by looking at various hydrocarbon indices that may explain or proffer solution to lack of discovery of commercial quantities of hydrocarbon within the basin (Ilozobhie, et al. 2014) and one of such indices is the Formation Temperature. The Formation Temperature is the temperature of the unperturbed formation within a basin. A critical understanding of the variation of Formation Temperature within an identified source rock formation as conceived in this research will go a long way to give an idea about the maturation of the source rock and in general, the hydrocarbon prospect of the Basin.

Generally,any prospective hydrocarbon basin must have a good quality source rock, good reservoir and seal lithologies, favourable regional pathways and trapping mechanisms Ayoola et al (1982) Ajayi and Ajakaiye (1983) Cratchley et al (1984) and Ilozobhie, et.al (2015). It is an established fact that the Fika Shale is one of the major source rock within the basin, while the reservoirs are provided by Gombe Sandstone and the Bima Sandstone facies of the basin. Also, the intrusive sills are known to be good seal for hydrocarbons while the aquifers within the Chad Formation can act as a conduit for hydrocarbon to the surface. Nwankwo and Ekine,(2009) and Ilozobhie et. al (2009). Thus the thrust of this study shall investigate the Formation Temperature distribution within the Turonian- Maastrichtian Fika Shale, in order to ascertain their effect on the hydrocarbon potential of the basin. 


\section{MATERIALS AND METHOD}

\subsection{Location of study Area}

The study area lies within an approximate location of latitude $12^{\circ} 09^{\prime} \mathrm{N}$ and $12^{\circ} 26^{\prime} \mathrm{N}$ and longitudes $15^{\circ} 12^{\prime} \mathrm{E}$ and $15^{\circ} 24^{\prime} \mathrm{E}$ and it covered an area of about 20 by 40 kilometer square of the total land mass of the entire basin (Fig. 1.0). In the concessional map of the basin, it is located in the oil prospecting lease (OPL) 721, 722, 732 and 733. The altitude of the basin dropped from $350 \mathrm{~m}$ at the western margin to about $300 \mathrm{~m}$ along a distance of roughly $240 \mathrm{~km}$ indicating a gentle slope towards lake Chad. The area of study is situated onshore and accessibility is by road and air.

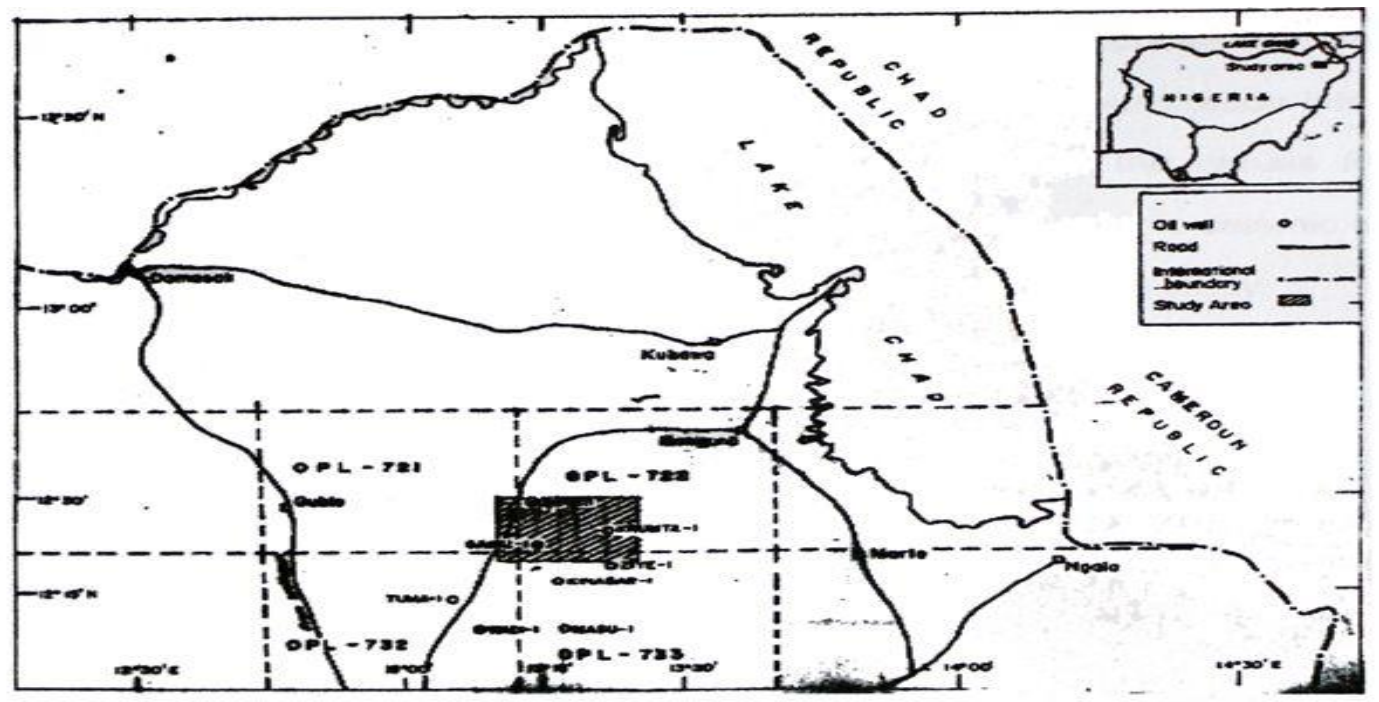

Fig. 1.0: Location map of Bornu Basin. Shaded section represent area covered in the study

\subsection{The Fika Shales of Borno Basin}

The Fika Shale is mainly dark to blue black shale overlying the Gongila Formation. These are completely marine shales consisting of blue black shales occasionally, gypsiferous with thin limestone intercalation. The thickness varies from about $1000 \mathrm{~m}$ in the NorthWestern margin to about 500m in the NorthEastern margin. Volcanic intrusive which occurs as diorites sills are present at several horizon of the formation (Okosun,1995). The unit is diachronous, dating from Turonian to Maastrichtian in age. The relative abundance of arenaceous benthic foraminifera within the Fika shale point to the prevalent of near shore environment. The Source of Marine water during the period is believed to be through the Tran Sahara sea way (Avbovbo et. al, 1986).

\subsection{Acquisition}

Soft copies of digital well data were supplied for this work. The soft copies of the well data given are Kinasar-1 ( $12^{0} 19^{\prime} \mathrm{N}$

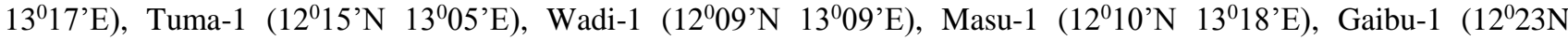
$\left.13^{0} 14^{\prime} \mathrm{E}\right)$, Krumta-1(12 $\left.26^{\prime} \mathrm{N} 13^{0} 23^{\prime} \mathrm{E}\right)$. This data were plotted by a developed visual basic plotter manager to facilitate the demarcation of bed boundaries and formations identification. Table (1.0) shows the wireline logs and the depth of investigation.

\subsection{Identification of the Fika Shale}

In this study, the gamma ray log signatures were utilized to delineate the sediments bed boundaries for the various formations (sandstone, shale and siltstone) encountered. Deductions from the electric logs show that this formation consists primarily of shale with minor limestone and subordinate amount of intercalated sand extending from $865 \mathrm{~m}$ to $1795 \mathrm{~m}$. Kinasar well reveals the Fika shale member from $865 \mathrm{~m}$ to $1250 \mathrm{~m}$ to be a shaly sequence with intercalations of sand $(865 m-975 m$ and $1045 m-1250 m)$. This strata is recognized by high Gamma ray values (except in the intercalated areas), high sonic travel time for shale zone but moderately low area of sands. A thick sequence of shale occurs between $1250 \mathrm{~m}$ to $1450 \mathrm{~m}$. It is recognized by high Gamma ray value and relatively high resistivity and high sonic travel time. Toward the end of the Fika Shale Formation $(1450 \mathrm{~m}$ to $1850 \mathrm{~m})$ there is a gradual increase in resistivity values with high gamma values with high sonic travel time compared to earlier zone. The log characteristics above indicate unconsolidated shale.

The Fika Shale was identified in the Krumta well within the depth range from 640m to $1990 \mathrm{~m}$ by the high Gamma ray values with high sonic travel time. The Intercalation sequence ranges from $640 \mathrm{~m}$ to $1110 \mathrm{~m}$. Most of the remaining 
section of the Fika shale depicts shaly sequence throughout ranging from $1110 \mathrm{~m}$ to $1990 \mathrm{~m}$ as reflected by high values of gamma ray, Resistivity and Sonic logs. The depth range of the Fika sequence identified within Masu, Tuma and Wadi ranges within $980 \mathrm{~m}$ to $1620 \mathrm{~m}, 700 \mathrm{~m}$ to $2710 \mathrm{~m}$ and $710 \mathrm{~m}$ to $2220 \mathrm{~m}$ respectively.

Table 1.0: Well location and wirelines log used for the study

\begin{tabular}{lllll}
\hline Well & $\begin{array}{l}\text { Drilling } \\
\text { depth } \\
\text { Range (m) }\end{array}$ & Lithology & Resistivity & Porosity \\
& & CAL. \& GR & & \\
Kinasar-1 & $45-4665$ & & SN \& ILD & $\Delta \mathrm{T}$ \& RHOB \\
Krumta-1 & $15-2950$ & CAL. \& GR & SN \& ILD & $\Delta \mathrm{T}$ \& RHOB \\
Masu-1 & $1996-3104$ & CAL. \& GR & MSFL \& & $\Delta \mathrm{T} \&$ RHOB \\
Tuma -1 & $33-3628$ & CAL. \& GR & SLD \& ILD & $\Delta \mathrm{T} \&$ RHOB \\
Wadi -1 & $539-3225$ & CAL. \& GR & SN \& ILD & $\Delta \mathrm{T} \&$ RHOB \\
\hline
\end{tabular}

$\begin{array}{lll}\text { Key: } & & \\ \text { CAL. } & & \text { Caliper log } \\ \text { SP } & = & \text { Spontaneous Potential Log } \\ \text { GR } & = & \text { Gamma Ray Log } \\ \text { SN } & = & \text { Short Normal Log } \\ \text { MSFL } & = & \text { Microspherical Focus Log } \\ \mathrm{ILD} & = & \text { Deep Induction Resistivity Log } \\ \Delta \mathrm{T} & = & \text { Sonic Log } \\ \text { RHOB } & = & \text { Density Log }\end{array}$

\subsection{Computation of Formation Temperature}

The computed formation temperature within each of the interested formation from each well was computed using the Borehole Temperature (BHT) values as displayed on the well header after which the BHT values were corrected. Several correction methods have been proposed by many authors ( Kutasov and Eppelbaum, 2003 and Onuoha and Ekine, 1999). In this study the BHT drilling effects were corrected by using an AAPG gradient correction factors (Table 2.0). The approach allows corrections to be made on individual recorded BHT data. The corrected BHT values were converted to the Formation Temperature using the Schlumberger correction chart (GEN-6). Table (3.0 to 8.0) shows the computed formation temperature for Fika shale at various coordinates and depths, this was done for the remaining four wells. Since all the given well data are from vertical well, the position of each of the well were posted on the corresponding depth point using the coordinate of such point to generates maps that shows the distribution of Formation Temperature within each well. Most importantly, in order to have an ideal about the variation of the Formation Temperature within the study the computed values were posted on the situation map using the coordinates of each depth points and are contoured using available contour software.

Table 2.0: Additions for correction of logged Bottom Hole Temperatures to True Formation Temperatures based on API method (Neglia, 1979).

\begin{tabular}{cc}
\hline $\begin{array}{c}\text { Depth } \\
(\mathbf{m})\end{array}$ & $\begin{array}{c}\text { Added } \\
\text { temperature } \\
\text { quotient }\left({ }^{\circ} \mathbf{C}\right)\end{array}$ \\
500 & 4.0 \\
1000 & 7.5 \\
1500 & 11.0 \\
2000 & 14.0 \\
2500 & 16.0 \\
3000 & 17.5 \\
3500 & 18.0 \\
4000 & 18.0 \\
4500 & 17.0 \\
5000 & 15.0 \\
5500 & 11.0 \\
6000 & 4.5 \\
\hline
\end{tabular}


Table 3: Computed Formation Temperature values with coordinates and depth in Kinasar well

Depth $(\mathbf{m})$


865
915
965
1015
1065
1115
1165
1215
1265
1315
1365
1415
1465
1515
1565
1615
1665
1715
1765
1795

Latitude

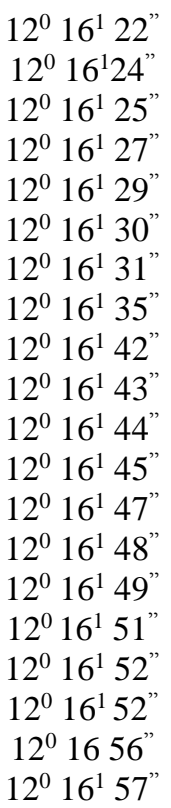

\section{Longitude}

$15^{0} 20^{1} 32^{\prime \prime}$

$15^{0} 20^{1} 33^{\prime \prime}$

$15^{0} 20^{1} 34^{\prime \prime}$

$15^{0} 20^{1} 35^{\prime \prime}$

$15^{0} 20^{1} 37^{\prime \prime}$

$15^{0} 20^{1} 38^{\prime \prime}$

$15^{0} 20^{1} 39^{\prime \prime}$

$15^{0} 20^{1} 42^{\prime \prime}$

$15^{0} 20^{1} 43^{\prime \prime}$

$15^{0} 20^{1} 46^{\prime \prime}$

$15^{0} 20^{1} 47^{\prime \prime}$

$15^{0} 20^{1} 49^{\prime \prime}$

$15^{0} 20^{1} 53^{\prime \prime}$

$15^{0} 20^{1} 55^{\prime \prime}$

$15^{0} 20^{1} 57^{\prime \prime}$

$15^{0} 20^{1} 58^{\prime \prime}$

$15^{0} 20^{1} 59^{\prime \prime}$

$15^{0} 21^{1} 01^{\prime \prime}$

$15^{0} 21^{1} 03^{\prime \prime}$

$15^{0} 21^{1} 04^{\prime \prime}$
Formation

Temperature within

Fika Formation

60

62

59

63

66

67

66

69

72

74

79

81

84

86

87

90

90

91

90

90

Table 4: The Formation Temperature values with depth in Krumta well

\begin{tabular}{|c|c|c|c|}
\hline $\operatorname{Depth}(\mathrm{m})$ & Latitude & Longitude & $\begin{array}{c}\text { Formation } \\
\text { Temperature } \\
\text { within Fika } \\
\text { Formation }\end{array}$ \\
\hline 640 & $12^{0} 16^{1} 16^{\prime \prime}$ & $15^{0} 19^{1} 52^{\prime \prime}$ & 65 \\
\hline 690 & $12^{0} 16^{1} 19^{\prime \prime}$ & $15^{0} 19^{1} 56^{\prime \prime}$ & 66 \\
\hline 740 & $12^{0} 16^{1} 20^{\prime \prime}$ & $15^{0} 20^{1} 03^{\prime \prime}$ & 59 \\
\hline 790 & $12^{0} 15^{1} 22^{\prime \prime}$ & $15^{0} 20^{1} 05^{\prime \prime}$ & 59 \\
\hline 840 & $12^{0} 16^{1} 23^{\prime \prime}$ & $15^{0} 20^{1} 08^{\prime \prime}$ & 62 \\
\hline 890 & $12^{0} 16^{1} 25^{\prime \prime}$ & $15^{0} 20^{1} 10^{\prime \prime}$ & 66 \\
\hline 940 & $12^{0} 16^{1} 26^{\prime \prime}$ & $15^{0} 20^{1} 13^{\prime \prime}$ & 66 \\
\hline 990 & $12^{0} 16^{1} 27^{\prime \prime}$ & $15^{0} 20^{1} 17^{\prime \prime}$ & 67 \\
\hline 1040 & $12^{0} 16^{1} 29^{\prime \prime}$ & $15^{0} 20^{1} 20^{\prime \prime}$ & 68 \\
\hline 1090 & $12^{0} 16^{1} 32^{\prime \prime}$ & $15^{0} 20^{1} 23^{\prime \prime}$ & 70 \\
\hline 1140 & $12^{0} 16^{1} 36^{\prime \prime}$ & $15^{0} 20^{1} 26^{\prime \prime}$ & 74 \\
\hline 1190 & $12^{0} 16^{1} 38^{\prime \prime}$ & $15^{0} 20^{1} 29^{\prime \prime}$ & 78 \\
\hline 1240 & $12^{0} 16^{1} 41^{\prime \prime}$ & $15^{0} 20^{1} 31^{\prime \prime}$ & 78 \\
\hline 1290 & $12^{0} 16^{1} 44^{\prime \prime}$ & $15^{0} 20^{1} 32^{\prime \prime}$ & 78 \\
\hline 1340 & $12^{0} 16^{1} 45^{\prime \prime}$ & $15^{0} 20^{1} 36^{\prime \prime}$ & 80 \\
\hline 1390 & $12^{0} 16^{1} 48^{\prime \prime}$ & $15^{0} 20^{1} 38^{\prime \prime}$ & 81 \\
\hline 1440 & $12^{0} 16^{1} 49^{\prime \prime}$ & $15^{0} 20^{1} 40^{\prime \prime}$ & 81 \\
\hline 1490 & $12^{0} 16^{1} 50^{\prime \prime}$ & $15^{0} 20^{1} 44^{\prime \prime}$ & 82 \\
\hline 1540 & $12^{0} 16^{\prime} 54^{\prime \prime}$ & $15^{0} 20^{1} 47^{\prime \prime}$ & 88 \\
\hline 1590 & $12^{0} 16^{1} 55^{\prime \prime}$ & $15^{0} 20^{1} 50^{\prime \prime}$ & 88 \\
\hline 1640 & $12^{0} 16^{1} 56^{\prime \prime}$ & $15^{0} 20^{1} 52^{\prime \prime}$ & 88 \\
\hline 1690 & $12^{0} 16^{1} 58^{\prime \prime}$ & $15^{0} 20^{1} 55^{\prime \prime}$ & 87 \\
\hline 1740 & $12^{0} 16^{\prime} 59^{\prime \prime}$ & $15^{0} 20^{1} 58^{\prime \prime}$ & 85 \\
\hline 1790 & $12^{0} 17^{1} 03^{\prime \prime}$ & $15^{0} 21^{1} 04^{\prime \prime}$ & 90 \\
\hline 1840 & $12^{0} 17^{1} 05^{\prime \prime}$ & $15^{0} 21^{1} 05^{\prime \prime}$ & 90 \\
\hline 1890 & $12^{0} 17^{1} 05^{\prime \prime}$ & $15^{0} 21^{1} 07^{\prime \prime}$ & 90 \\
\hline 1940 & $12^{0} 17^{1} 07^{\prime \prime}$ & $15^{0} 21^{1} 10^{\prime \prime}$ & 90 \\
\hline 1990 & $12^{0} 17^{1} 09^{\prime \prime}$ & $15^{0} 21^{1} 03^{\prime \prime}$ & 90 \\
\hline
\end{tabular}


Table 5: The Formation Temperature values with depth in Masu well

\begin{tabular}{cccc} 
Depth(m) & Latitude & Longitude & $\begin{array}{c}\text { Formation } \\
\text { Temperature } \\
\text { within Fika } \\
\text { Formation }\end{array}$ \\
980 & $12^{0} 16^{1} 27^{\prime \prime}$ & $15^{0} 20^{1} 24^{\prime \prime}$ & 58 \\
1030 & $12^{0} 16^{1} 30^{\prime \prime}$ & $15^{0} 20^{1} 27^{\prime \prime}$ & 59 \\
1080 & $12^{0} 16^{1} 33^{\prime \prime}$ & $15^{0} 20^{1} 29^{\prime \prime}$ & 63 \\
1130 & $12^{0} 16^{1} 36^{\prime \prime}$ & $15^{0} 20^{1} 30^{\prime \prime}$ & 62 \\
1180 & $12^{0} 16^{1} 39^{\prime \prime}$ & $15^{0} 20^{1} 32^{\prime \prime}$ & 66 \\
1230 & $12^{0} 16^{1} 42^{\prime \prime}$ & $15^{0} 20^{1} 36^{\prime \prime}$ & 69 \\
1280 & $12^{0} 16^{1} 43^{\prime \prime}$ & $15^{0} 20^{1} 38^{\prime \prime}$ & 72 \\
1330 & $12^{0} 16^{1} 46^{\prime \prime}$ & $15^{0} 20^{1} 40^{\prime \prime}$ & 74 \\
1380 & $12^{0} 16^{1} 49^{\prime \prime}$ & $15^{0} 20^{1} 42^{\prime \prime}$ & 79 \\
1430 & $12^{0} 16^{1} 50^{\prime \prime}$ & $15^{0} 20^{1} 44^{\prime \prime}$ & 85 \\
1480 & $12^{0} 16^{1} 52^{\prime \prime}$ & $15^{0} 20^{1} 48^{\prime \prime}$ & 87 \\
1530 & $12^{0} 16^{1} 53^{\prime \prime}$ & $15^{0} 20^{1} 51^{\prime \prime}$ & 90 \\
1580 & $12^{0} 16^{1} 54^{\prime \prime}$ & $15^{0} 20^{1} 52^{\prime \prime}$ & 90 \\
1620 & $12^{0} 16^{1} 54^{\prime \prime}$ & $15^{0} 20^{1} 55^{\prime \prime}$ & 90 \\
\hline
\end{tabular}


Table 6: The Formation Temperature values with depth in Tuma well

\begin{tabular}{|c|c|c|c|}
\hline $\operatorname{Depth}(\mathrm{m})$ & Latitude & Longitude & $\begin{array}{c}\text { Formation } \\
\text { Temperature } \\
\text { within Fika } \\
\text { Formation }\end{array}$ \\
\hline 700 & $12^{0} 16^{1} 23^{\prime \prime}$ & $15^{0} 19^{1} 56^{\prime \prime}$ & 60 \\
\hline 750 & $12^{0} 16^{1} 27^{\prime \prime}$ & $15^{0} 19^{1} 58^{\prime \prime}$ & 60 \\
\hline 800 & $12^{0} 16^{1} 29^{\prime \prime}$ & $15^{0} 20^{1} 00^{\prime \prime}$ & 62 \\
\hline 850 & $12^{0} 16^{1} 32^{\prime \prime}$ & $15^{0} 20^{1} 03^{\prime \prime}$ & 61 \\
\hline 900 & $12^{0} 16^{1} 34^{\prime \prime}$ & $15^{0} 20^{1} 06^{\prime \prime}$ & 63 \\
\hline 950 & $12^{0} 16^{1} 35^{\prime \prime}$ & $15^{0} 20^{1} 06^{\prime \prime}$ & 66 \\
\hline 1000 & $12^{0} 16^{1} 37^{\prime \prime}$ & $15^{0} 20^{1} 08^{\prime \prime}$ & 67 \\
\hline 1050 & $12^{0} 16^{1} 39^{\prime \prime}$ & $15^{0} 20^{1} 09^{\prime \prime}$ & 67 \\
\hline 1100 & $12^{0} 16^{1} 40^{\prime \prime}$ & $15^{0} 20^{1} 12^{\prime \prime}$ & 69 \\
\hline 1150 & $12^{0} 16^{1} 43^{\prime \prime}$ & $15^{0} 20^{1} 15^{\prime \prime}$ & 69 \\
\hline 1200 & $12^{0} 16^{1} 44^{\prime \prime}$ & $15^{0} 20^{1} 18^{\prime \prime}$ & 70 \\
\hline 1250 & $12^{0} 16^{1} 46^{\prime \prime}$ & $15^{0} 20^{1} 22^{\prime \prime}$ & 72 \\
\hline 1300 & $12^{0} 16^{1} 47^{\prime \prime}$ & $15^{0} 20^{1} 23^{\prime \prime}$ & 69 \\
\hline 1350 & $12^{0} 16^{1} 48^{\prime \prime}$ & $15^{0} 20^{1} 26^{\prime \prime}$ & 69 \\
\hline 1400 & $12^{0} 16^{1} 52^{\prime \prime}$ & $15^{0} 20^{1} 27^{\prime \prime}$ & 70 \\
\hline 1450 & $12^{0} 16^{1} 53^{\prime \prime}$ & $15^{0} 20^{1} 29^{\prime \prime}$ & 75 \\
\hline 1500 & $12^{0} 16^{1} 55^{\prime \prime}$ & $15^{0} 20^{1} 32 "$ & 75 \\
\hline 1550 & $12^{0} 16^{1} 56^{\prime \prime}$ & $15^{0} 20^{1} 35^{\prime \prime}$ & 75 \\
\hline 1600 & $12^{0} 16^{1} 58^{\prime \prime}$ & $15^{0} 20^{1} 38^{\prime \prime}$ & 75 \\
\hline 1650 & $12^{0} 17^{1} 02^{\prime \prime}$ & $15^{0} 20^{1} 40^{\prime \prime}$ & 74 \\
\hline 1700 & $12^{0} 17^{1} 04^{\prime \prime}$ & $15^{0} 20^{1} 43^{\prime \prime}$ & 75 \\
\hline 1750 & $12^{0} 17^{1} 07^{\prime \prime}$ & $15^{0} 20^{1} 47^{\prime \prime}$ & 75 \\
\hline 1800 & $12^{0} 17^{1} 10^{\prime \prime}$ & $15^{0} 20^{1} 49^{\prime \prime}$ & 75 \\
\hline 1850 & $12^{0} 17^{1} 14^{\prime \prime}$ & $15^{0} 20^{1} 53^{\prime \prime}$ & 75 \\
\hline 1900 & $12^{0} 17^{1} 16^{\prime \prime}$ & $15^{0} 20^{1} 56^{\prime \prime}$ & 76 \\
\hline 1950 & $12^{0} 17^{1} 19^{\prime \prime}$ & $15^{0} 21^{1} 01^{\prime \prime}$ & 77 \\
\hline 2000 & $12^{0} 17^{1} 22^{\prime \prime}$ & $15^{0} 21^{1} 03^{\prime \prime}$ & 79 \\
\hline 2050 & $12^{0} 17^{1} 25^{\prime \prime}$ & $15^{0} 21^{1} 06^{\prime \prime}$ & 79 \\
\hline 2100 & $12^{0} 17^{1} 26^{\prime \prime}$ & $15^{0} 21^{1} 08^{\prime \prime}$ & 79 \\
\hline 2150 & $12^{0} 17^{1} 28^{\prime \prime}$ & $15^{0} 21^{1} 08^{\prime \prime}$ & 79 \\
\hline 2200 & $12^{0} 17^{1} 30^{\prime \prime}$ & $15^{0} 21^{1} 11^{\prime \prime}$ & 79 \\
\hline 2250 & $12^{0} 17^{1} 32^{\prime \prime}$ & $15^{0} 21^{1} 13^{\prime \prime}$ & 79 \\
\hline 2300 & $12^{0} 17^{1} 34^{\prime \prime}$ & $15^{0} 21^{1} 15^{\prime \prime}$ & 79 \\
\hline 2350 & $12^{0} 17^{1} 36^{\prime \prime}$ & $15^{0} 21^{1} 17^{\prime \prime}$ & 82 \\
\hline 2400 & $12^{0} 17^{1} 38^{\prime \prime}$ & $15^{0} 21^{1} 19^{\prime \prime}$ & 82 \\
\hline 2450 & $12^{0} 17^{1} 41^{\prime \prime}$ & $15^{0} 21^{1} 21^{\prime \prime}$ & 83 \\
\hline 2500 & $12^{0} 17^{1} 44^{\prime \prime}$ & $15^{0} 21^{1} 23^{\prime \prime}$ & 86 \\
\hline 2550 & $12^{0} 17^{1} 47^{\prime \prime}$ & $15^{0} 21^{1} 26^{\prime \prime}$ & 89 \\
\hline 2600 & $12^{0} 17^{1} 48^{\prime \prime}$ & $15^{0} 21^{1} 28^{\prime \prime}$ & 90 \\
\hline 2650 & $12^{0} 17^{1} 48^{\prime \prime}$ & $15^{0} 21^{1} 28^{\prime \prime}$ & 87 \\
\hline 2700 & $12^{0} 17^{1} 51^{\prime \prime}$ & $15^{0} 21^{1} 31^{\prime \prime}$ & 90 \\
\hline 2710 & $12^{0} 17^{1} 51^{\prime \prime}$ & $15^{0} 21^{1} 31^{\prime \prime}$ & 90 \\
\hline
\end{tabular}


Table 7: The Formation Temperature values with depth in Wadi well

\begin{tabular}{|c|c|c|c|}
\hline $\operatorname{Depth}(\mathrm{m})$ & Latitude & Longitude & $\begin{array}{c}\text { Formation } \\
\text { Temperature } \\
\text { within Fika } \\
\text { Formation }\end{array}$ \\
\hline 710 & $12^{0} 16^{1} 22^{\prime \prime}$ & $15^{0} 19^{1} 53^{\prime \prime}$ & 62 \\
\hline 760 & $12^{0} 16^{1} 23^{\prime \prime}$ & $15^{0} 19^{1} 55^{\prime \prime}$ & 62 \\
\hline 810 & $12^{0} 16^{1} 24^{\prime \prime}$ & $15^{0} 19^{1} 56^{\prime \prime}$ & 63 \\
\hline 860 & $12^{0} 16^{1} 26^{\prime \prime}$ & $15^{0} 19^{1} 59^{\prime \prime}$ & 61 \\
\hline 910 & $12^{0} 16^{1} 27^{\prime \prime}$ & $15^{0} 20^{1} 03^{\prime \prime}$ & 65 \\
\hline 960 & $12^{0} 16^{1} 29^{\prime \prime}$ & $15^{0} 20^{1} 06^{\prime \prime}$ & 65 \\
\hline 1010 & $12^{0} 16^{1} 32^{\prime \prime}$ & $15^{0} 20^{1} 09^{\prime \prime}$ & 65 \\
\hline 1060 & $12^{0} 16^{1} 36^{\prime \prime}$ & $15^{0} 20^{1} 12^{\prime \prime}$ & 67 \\
\hline 1110 & $12^{0} 16^{1} 39^{\prime \prime}$ & $15^{0} 20^{1} 15^{\prime \prime}$ & 66 \\
\hline 1160 & $12^{0} 16^{1} 41^{\prime \prime}$ & $15^{0} 20^{1} 18^{\prime \prime}$ & 64 \\
\hline 1210 & $12^{0} 16^{1} 44^{\prime \prime}$ & $15^{0} 20^{1} 20^{\prime \prime}$ & 66 \\
\hline 1260 & $12^{0} 16^{1} 47^{\prime \prime}$ & $15^{0} 20^{1} 23^{\prime \prime}$ & 68 \\
\hline 1310 & $12^{0} 16^{1} 48^{\prime \prime}$ & $15^{0} 20^{1} 25^{\prime \prime}$ & 72 \\
\hline 1360 & $12^{0} 16^{1} 50^{\prime \prime}$ & $15^{0} 20^{1} 27^{\prime \prime}$ & 73 \\
\hline 1410 & $12^{0} 16^{1} 52^{\prime \prime}$ & $15^{0} 20^{1} 28^{\prime \prime}$ & 76 \\
\hline 1460 & $12^{0} 16^{1} 53^{\prime \prime}$ & $15^{0} 20^{1} 30^{\prime \prime}$ & 76 \\
\hline 1510 & $12^{0} 16^{1} 56^{\prime \prime}$ & $15^{0} 20^{1} 33^{\prime \prime}$ & 78 \\
\hline 1560 & $12^{0} 16^{1} 58^{\prime \prime}$ & $15^{0} 20^{1} 36^{\prime \prime}$ & 80 \\
\hline 1610 & $12^{0} 16^{1} 59^{\prime \prime}$ & $15^{0} 20^{1} 38^{\prime \prime}$ & 85 \\
\hline 1660 & $12^{0} 17^{1} 02^{\prime}$ & $15^{0} 20^{1} 41^{\prime \prime}$ & 84 \\
\hline 1710 & $12^{0} 17^{1} 07^{\prime \prime}$ & $15^{0} 20^{1} 42^{\prime}$ & 85 \\
\hline 1760 & $12^{0} 17^{1} 09 "$ & $15^{0} 20^{1} 44^{\prime \prime}$ & 85 \\
\hline 1810 & $12^{0} 17^{1} 13^{\prime \prime}$ & $15^{0} 20^{1} 46^{\prime \prime}$ & 87 \\
\hline 1860 & $12^{0} 17^{1} 12^{\prime}$ & $15^{0} 20^{1} 46^{\prime \prime}$ & 87 \\
\hline 1910 & $12^{0} 17^{1} 15^{\prime \prime}$ & $15^{0} 20^{1} 49^{\prime \prime}$ & 78 \\
\hline 1960 & $12^{0} 17^{1} 17^{\prime \prime}$ & $15^{0} 20^{1} 52^{\prime \prime}$ & 79 \\
\hline 2010 & $12^{0} 17^{1} 19^{\prime \prime}$ & $15^{0} 20^{1} 54^{\prime \prime}$ & 78 \\
\hline 2060 & $12^{0} 17^{1} 22^{\prime \prime}$ & $15^{0} 20^{1} 58^{\prime \prime}$ & 78 \\
\hline 2110 & $12^{0} 17^{1} 24 "$ & $15^{0} 21^{1} 02^{\prime \prime}$ & 78 \\
\hline 2160 & $12^{0} 17^{1} 27^{\prime \prime}$ & $15^{0} 21^{1} 06^{\prime \prime}$ & 79 \\
\hline 2210 & $12^{0} 17^{1} 30^{\prime \prime}$ & $15^{0} 21^{1} 10^{\prime \prime}$ & 78 \\
\hline 2220 & $12^{0} 17^{1} 30^{\prime \prime}$ & $15^{0} 21^{1} 11^{\prime \prime}$ & 78 \\
\hline
\end{tabular}

Table 8:The Formation Temperature values with depth in Ziye well

\begin{tabular}{cccc}
\hline Depth(m) & Latitude & Longitude & $\begin{array}{c}\text { Formation } \\
\text { Temperature } \\
\text { within Fika } \\
\text { Formation }\end{array}$ \\
790 & $12^{0} 16^{1} 20^{\prime \prime}$ & $15^{0} 19^{1} 55^{\prime \prime}$ & 65 \\
840 & $12^{0} 16^{1} 23^{\prime \prime}$ & $15^{0} 19^{1} 59^{\prime \prime}$ & 65 \\
890 & $12^{0} 16^{1} 24^{\prime \prime}$ & $15^{0} 20^{1} 02^{\prime \prime}$ & 65 \\
940 & $12^{0} 16^{1} 27^{\prime \prime}$ & $15^{0} 20^{1} 04^{\prime \prime}$ & 72 \\
990 & $12^{0} 16^{1} 30^{\prime \prime}$ & $15^{0} 20^{1} 07^{\prime \prime}$ & 68 \\
1040 & $12^{0} 16^{1} 32^{\prime \prime}$ & $15^{0} 20^{1} 09^{\prime \prime}$ & 76 \\
1090 & $12^{0} 16^{1} 34^{\prime \prime}$ & $15^{0} 20^{1} 11^{\prime \prime}$ & 77 \\
1140 & $12^{0} 16^{1} 38^{\prime \prime}$ & $15^{0} 20^{1} 14^{\prime \prime}$ & 82 \\
1190 & $12^{0} 16^{1} 40^{\prime \prime}$ & $15^{0} 20^{1} 17^{\prime \prime}$ & 82 \\
1240 & $12^{0} 16^{1} 43^{\prime \prime}$ & $15^{0} 20^{1} 19^{\prime \prime}$ & 86 \\
1290 & $12^{0} 16^{1} 46^{\prime \prime}$ & $15^{0} 20^{1} 22^{\prime \prime}$ & 88 \\
1340 & $12^{0} 16^{1} 49^{\prime \prime}$ & $15^{0} 20^{1} 24^{\prime \prime}$ & 88 \\
1390 & $12^{0} 16^{1} 52 "$ & $15^{0} 20^{1} 27^{\prime \prime}$ & 90 \\
\hline
\end{tabular}




\section{RESULTS AND DISCUSSION}

\subsection{Kinasar Well}

The Formation Temperature (FT) within this field ranges within $30^{\circ} \mathrm{C}$ to $76^{\circ} \mathrm{C}$ (Fig. 2.0) the temperature variation is gentle toward the South Eastern region, with the North - Eastern part of the well is much steeper, having high Formation temperature ranging between $64^{\circ} \mathrm{C}$ to $76^{\circ} \mathrm{C}$, the North Western part displace temperature range between $44^{\circ} \mathrm{C}$ to $58^{\circ} \mathrm{C}$. The distribution of the temperature is along the South Western - North Eastern direction with the central part showing a range between $54^{\circ} \mathrm{C}$ to $62^{\circ} \mathrm{C}$.

\subsection{Krumta Well}

The outward steepy spreading of the temperature values from the westward side of the study area is clearly seen on this map likely indicating rapid variation in geothermal. The North - Eastern part showing relatively high Formation Temperature from $85^{\circ} \mathrm{C}$ to $100^{\circ} \mathrm{C}$ (Fig. 3.0). The North Western part ranges from $50^{\circ} \mathrm{C}$ to $90^{\circ} \mathrm{C}$. The central part shows

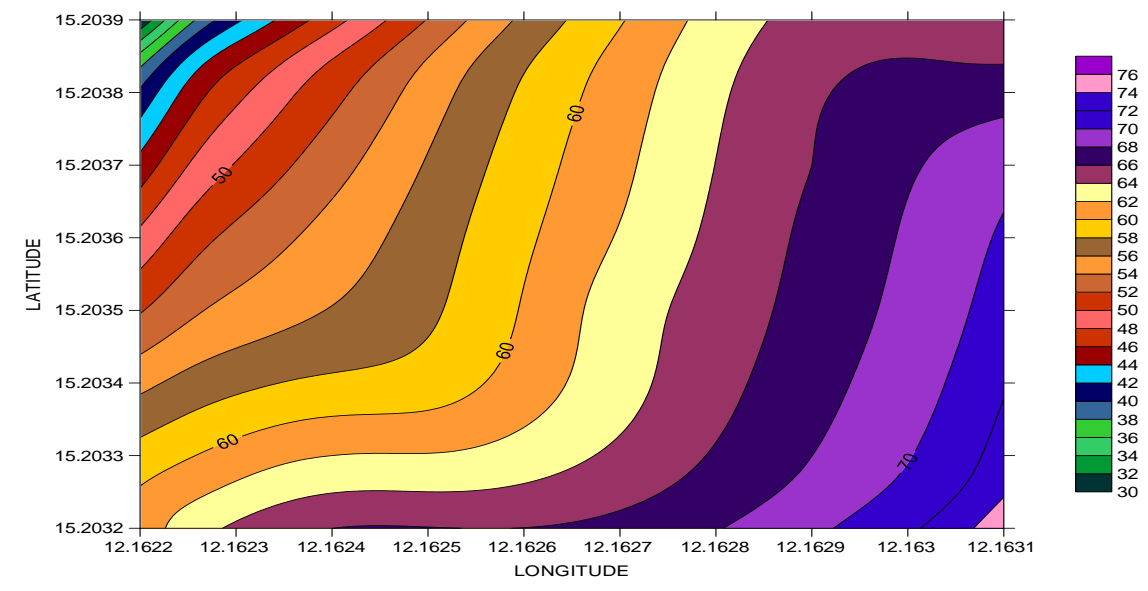

Fig. 2.0: Formation temperature map within Fika Formation in Kinasar well

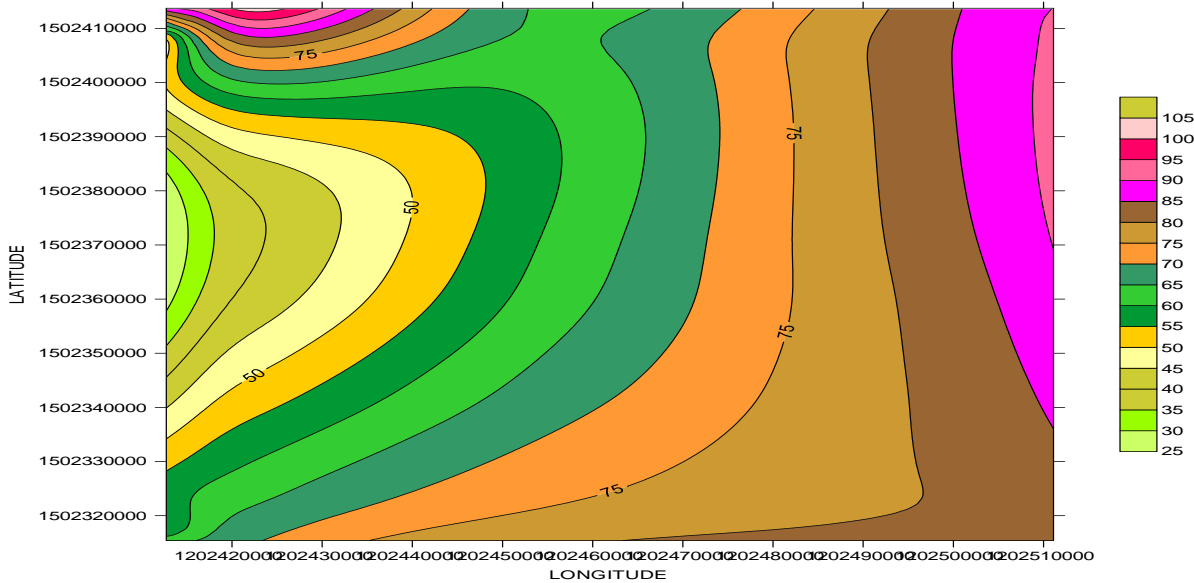

Fig.3.0: Formation temperature map within fika formation in Krumta well

relatively low temperature distribution from $55^{\circ} \mathrm{C}$ to $70^{\circ} \mathrm{C}$.The distribution still maintain the North Eastern - South Western pattern.

\subsection{Masu Well}

The Temperature of the Formation ranging within $56^{\circ} \mathrm{C}$ to $108^{\circ} \mathrm{C}$. The well exhibit evenly distribution of temperature within the South central region (Fig.4.0). The Upper part (North Eastern) shows high formation Temperature values ranging within $86^{\circ} \mathrm{C}$ to $108^{\circ} \mathrm{C}$. The direction the Temperature is trending is still South Western North Eastern direction. 


\subsection{Tuma Well}

The Formation Temperature ranges from $80^{\circ} \mathrm{C}$ to $195^{\circ} \mathrm{C}$ trending South Western - North Eastern direction with the Central region having Formation Temperature ranging from $120^{\circ} \mathrm{C}$ to $130^{\circ} \mathrm{C}$ and the North Eastern part temperature values between $160^{\circ} \mathrm{C}$ to $165^{\circ} \mathrm{C}$ while the South Eastern part depicts high temperature values within $175^{\circ} \mathrm{C}$ to $195^{\circ} \mathrm{C}$ (Fig.5.0)
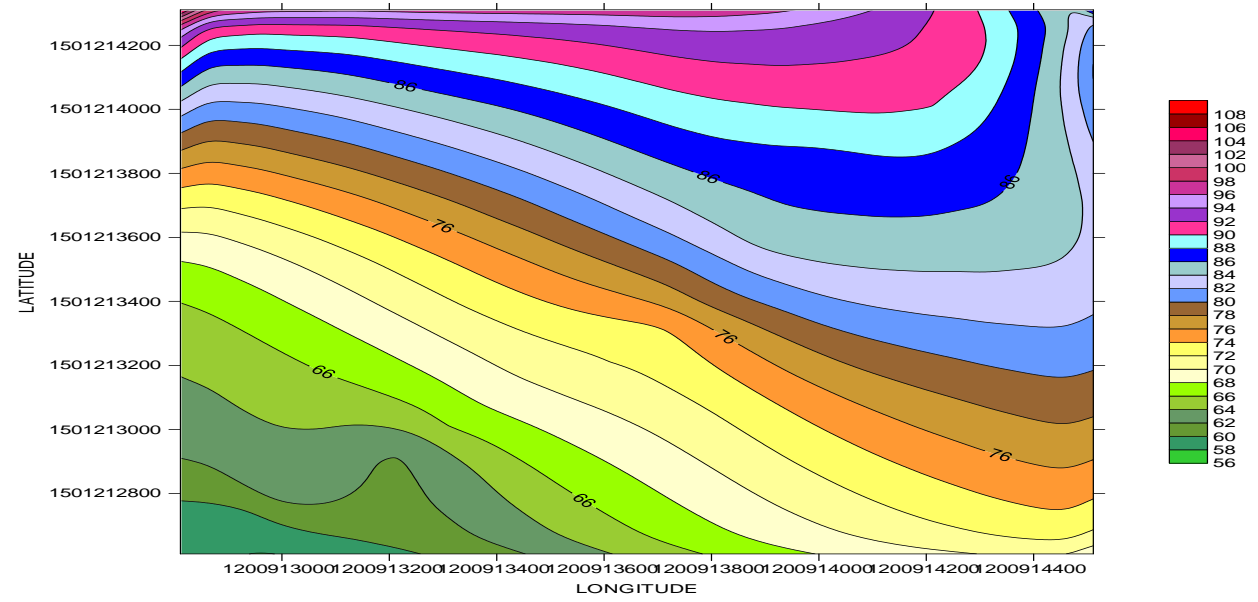

Fig.4.0: Formation temperature map within fika formation in Masu well

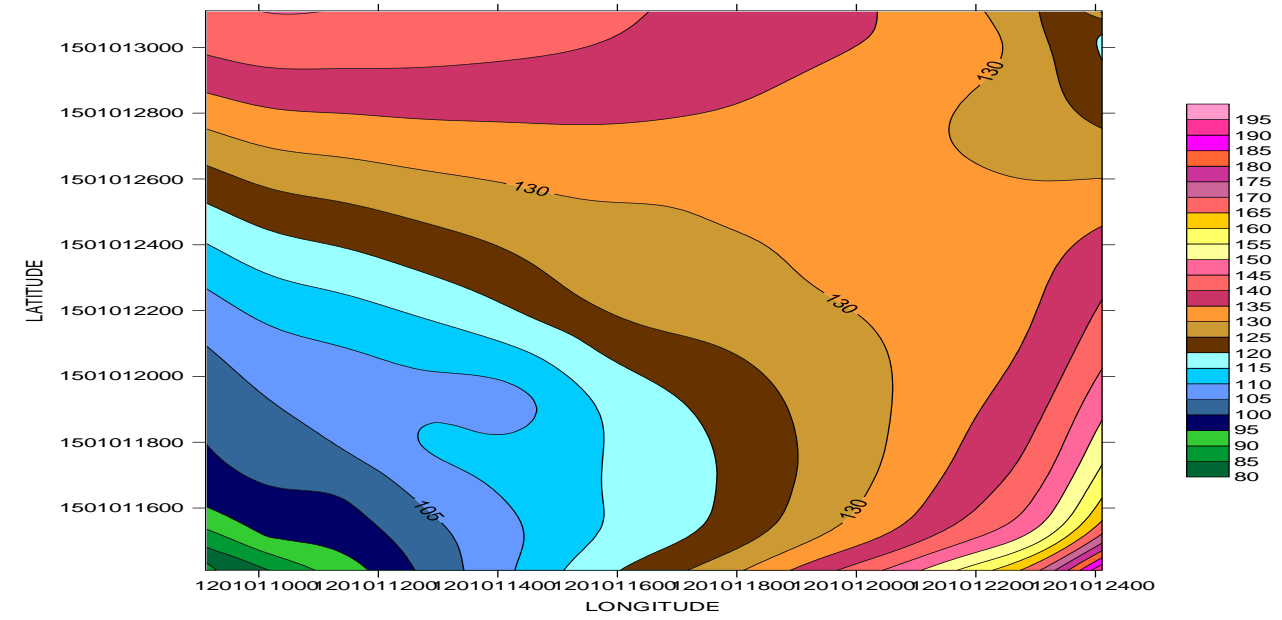

Fig. 5.0: Formation temperature map within fika formation in Tuma well

\subsection{Wadi Well}

There is a progressive build up of the formation temperature in the South Western - North Eastern direction with the Southern part clearly showing this build up (Fig.6.0). The South Eastern part shows high temperature values between $160^{\circ} \mathrm{C}$ to $210^{\circ} \mathrm{C}$ while the central portion has relatively low temperature values from $120^{\circ} \mathrm{C}$ to $145^{\circ} \mathrm{C}$.

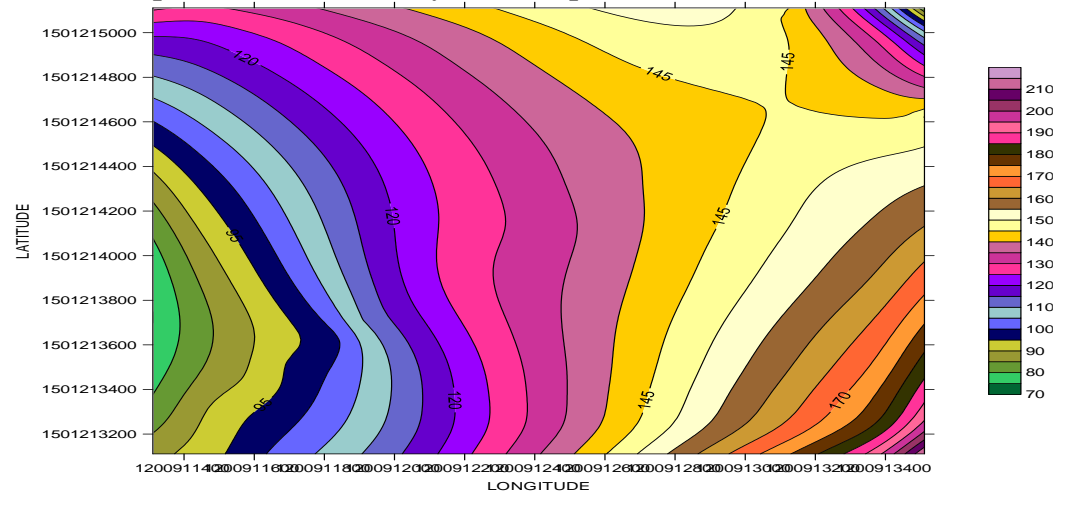

Fig.6.0: Formation temperature map within Fika formation in Wadi well 


\subsection{Formation Temperature analysis within the study area}

A critical investigation of the map of all the wellbore temperatures shows remarkable steep variations in temperature with increased range from $68^{\circ} \mathrm{C}$ to $128^{\circ} \mathrm{C}$ starting from the southern eastern region to the north western region (Fig.7.0). This is possibly due to substantial temperature enhancing effects of the underlying basement complex. Interestingly, it was also observed that the minimal temperature variation occurred with approximately $2^{\circ} \mathrm{C} /$ meter across the field and this also lays credence to the fact that the notable subsurface geothermal variation may be recent events initiated by the near- surface magmatic events which may have had adverse effects on the overlying sedimentary cover.

The north east to south west region showed diagonally uniform cross sectional temperatures ranging from $90^{\circ} \mathrm{C}$ to $102^{\circ} \mathrm{C}$. This suggests that possible hydrocarbon potential occurrences in this field may have better prospects from the central region with an average temperature of $98^{\circ} \mathrm{C}$ to the south eastern region with the least temperature of $68^{\circ} \mathrm{C}$ as long as favourable sedimentary covers exist. Furthermore, it is suggested that the probability of hydrocarbon is better in the south eastern region than the north western region although the steep temperature variation of $2^{\circ} \mathrm{C} /$ meter may perhaps reduce this possibility.

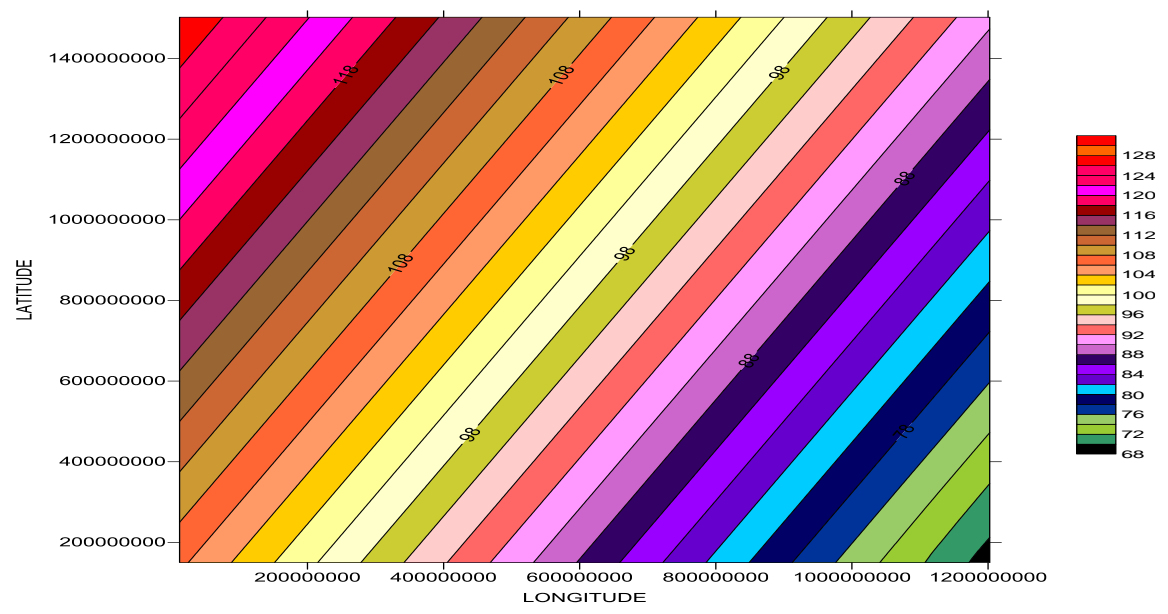

Fig. 7.0: Formation temperature map within the study area

\section{CONCLUSION}

The formation Temperature distribution within Fika Shale source rock of the Bornu basin was investigated. The linear increase of temperature with depth was evident throughout the study area within each well especially within Krumta and Kinasar wells while Masu, Tuma and Wadi shows high formation temperature variation with depth. The spatial variation of Formation Temperature clearly shows that some area (the south western region to the north eastern region) depicts steepy variations in temperature $\left(68^{\circ} \mathrm{C}\right.$ to $\left.128^{\circ} \mathrm{C}\right)$ this is attributed possibly to substantial magmatic intrusive bodies which the basin is noted for within this region this may be responsible for non availability of hydrocarbon within the basin since the formation temperature within this region is very high for liquid hydrocarbon to accumulate.

\section{REFERENCES}

- $\quad$ Ajayi, C. I \& Ajakaiye, D.E. (1983). The Origin and Pecularities of the Nigerian Benue Trough: Another look from recent gravity data obtained from middle Benue. Tectonphysics, Vol. 10(80), pp. 285-303.

- Avbovbo, A. A, Eyoola, E. O. \& Osahon G. A. (1986). Depositional and structural styles in Chad basin of Northeastern Nigeria. American Association of Petroleum Geologists Bulletin. 80, pp. 1787-1798.

- Ayoola E.O. Amaechi, M. \& Chukwu, R. (1982). Nigeria's Newer petroleum Exploration provinces Benue, Chad and Sokoto Basins. Journal of Mining and Geology. Vol. 19(1), pp. 72- 87.

- Cratchley, C. R. Louis, P. \& Ajakaiye, D. E. (1984). Geophysical and geological evidence for the Benue - Chad Basin Cretaceous rift valley system and its technique implications. Journal of African Earth Sciences. Vol. 6(2), pp. 141- 150 . 
- Ilozobhie, A.J. Okwueze, E .E \& Egeh, E.U (2009). Sand - shaliness evaluation of part of Bornu basin using well log data. Nigerian Journal of Physics Vol. 21(1), pp. 11- 19.

- Ilozobhie, A.J, Obi, D.A Okwueze, E.E and Okereke C.S (2014) Geophysical studies of part of Bornu Basin from Seismic, Well log and Aeromagnetic data. World Journal of Applied Science and Technology Vol. 6 (2) pp105-113

- Ilozobhie, A.J. Obi, D.A \& Okwueze, E.E (2015). Geostatistical analysis of porosity distribution from well log data, in part of Bornu Basin, North- eastern part, Nigeria using Kriging and Co- Kriging methods. Advances in Applied Science Research Vol. 6(2) pp.83-95.

- Kutasov I.M. \& Eppelbaum L.V. (2003). A new method for determining the formation temperature from bottom-hole temperature logs. Journal of Petroleum and Gas Engineering Vol. 1(1), pp. 1-8.

- Neglia, S (1979). Migration of fluids in sedimentary basins. America Association of Petroleum Geologists Bulletin. 63, pp. $573-597$.

- Nwankwo C.N. \& Ekine,A.S. (2009). Geothermal gradients in the Chad Basin, Nigeria, from bottom hole temperature logs. International Journal of Physical Sciences Vol. 4 (12), pp. 777-783.

- Okosun, A. E. (1995). Review of the geology of Bornu Basin. Journal of Mining and Geology. Vol. 31 (2), pp. $112-113$

- Onuoha, K. N \& Ekine, A.S (1999). Subsurface temperature variations and heat flow in the Anambra basin, Nigeria Journal Africa Earth ScienceVol. 28(3), pp. 641 - 652. 